\title{
Stimulating innovation on social product development: an analysis of social behaviors in online innovation communities
}

\author{
Chunjia Han and Mu Yang \\ Department of Systems Management and Strategy, University of Greenwich, UK
}

\begin{abstract}
With firms' increasing adoption of social product development strategies, such as mass collaboration and crowdsourcing, online users are actively participating in the development of new products and services via social media platforms. Online innovation communities (OICs), one representative of such social media platforms, have been used by large firms to collect ideas from their users and facilitate the product development process. While it is extensively studied that product ideas with high popularity on OICs are of great importance to the product development, research on what social behaviors of online users lead to the high popularity is largely unclear. This paper conducts an empirical study by collecting a large-scale, quantitative dataset from an OIC between 2014 and 2018. With the analysis of users' online idea posting and commenting behaviors, our results reveal that the idea contribution experience, together with comment diversity positively influence the overall popularity of an individual's ideas, while the motivation of providing comments is negatively related. Moreover, user's innovation capability poses a positive effect on both overall and average popularity of ideas. These findings can help firms better incentivise their users on OICs to improve the effectiveness and efficiency of social product development.
\end{abstract}

Managerial relevance statement: This study has significant managerial implications. It provides guidance to firms on how to make use of idea popularity in OICs 
in order to stimulate innovation on social product development. Our research demonstrates that idea popularity is an efficient and effective measure for idea pre-selection. Considering the huge number of ideas generated in OICs, the findings of the study would help firms save effort and time on idea evaluation significantly. However, as OICs are social media platforms, firms have concerns that voting behaviors might be affected by users' social networks, which makes it inappropriate to use voted popularity as the idea pre-selection mechanism. Our study tackled these research problems by disclosing that an ideator's social behaviors are related to idea popularity. Furthermore, by contributing to the research area of lead users' characteristics, this study creates a fresh angle for firms to better identify their lead users. Finally, the findings on the relation between social behaviors and idea creation generate valuable suggestions to firms on how OICs should be structured and managed in order to stimulate the production of qualified ideas.

\section{Introduction}

The use of external individuals, particularly customers, as participants in problem solving can benefit firms innovation, particularly in the success of new product development (NPD) [6]. Although developing new products is an important innovation activity for firms, the failure rate of newly introduced products in market is as high as 40 percent [7]. One of the reasons for the high failure rate is that it is hard to predict customers' needs and what they are willing to buy. To reduce the risks and uncertainties, firms are suggested to get their customers involved into the process of NPD and the ideation phase [8]. Thanks to the development of social computing technologies, it is possible for customers to become not only involved, but socially engaged in NPD. This becomes essential to strengthen customers' active roles in NPD. One example of this socially-engaged NPD supported by social computing technologies is crowdsourcing,

which is widely applied in the development of open source software. Crowdsourcing 
is "the act of taking a job traditionally performed by a designated agent (usually an employee) and outsourcing it to an undefined, generally large group of people in the form of an open call" [9]. Relying on social computing technologies makes product innovation more efficient and effective. Moreover, the application of social computing technologies creates a new model, which "extends open innovation beyond customerinvolvement models to socially-engaged individual actors fully involved in ideation and development of new products" [10]. The new model is commonly called social product development.

Social product development emphasizes that firms should not only involve people from internally and externally, but also socially engage them with innovation process [11]. In the model of social product development, a qualified crowd of users is encouraged to influence the product life cycle at any stage via social communication and collaboration. The advantage of social product development is that, innovation projects which are developed as a result benefit from shorter development time and less investment compaired with similar projects based solely on internal research [12]. This is because getting a crowd of users socially engaged into NPD can improve the efficiency of communication and collaboration in the innovation process. Social product development enhances efficiency of communication and collaboration through the application of social computing techniques such as social networking sites and online communities [11].

Online communities work as a channel to actively connect firms with people from both internal and external sources. Valuable information can be generated by people via posting and commenting activities in online communities. Firms then use online communities to collect information, and develop communication and collaboration; different types of online communities are set up by firms with various purposes. There are some online communities developed in order to collect users' new ideas and suggestions on firms' products and services, these are known as online innovation communities (OICs). Widely known examples of OICs include Dell's IdeaStorm and Starbucks' 
MyStarbucksIdea. They are online communities in which customers are encouraged to post their new ideas and suggestions about Dell's and Starbucks' products and services. According to the data published, both IdeaStorm and MyStarbucksIdea have successfully collected valuable information from external crowd. A significant number of collected new ideas have been employed by Dell and Starbucks [1]. IdeaStorm and MyStarbucksIdea are not the only examples for the application of OICs. In fact, it has been reported that firms, in particular high-tech firms, which have widely established OICs can benefit from customers' inputs. $80 \%$ of high-tech firms listed in the S\&P 500 index have launched OICs in their business [13].

Thousands of users have been attracted to actively participate in OICs, they produce a huge number of new ideas, which contribute to the development of products and services. Moreover, OICs make it possible to let a crowd of users participate in the review of new ideas posted in OICs. People are encouraged to vote, comment and discuss about new ideas launched by others. This becomes important for the success of OICs, as it not only helps to improve the quality of new ideas via peer discussion, but also helps to identify the popularity of new ideas from the masses. Obviously, identifying popular ideas is important for NPD, because it reflects a crowd of users' opinions on their demands [1]. In other words, the popularity of new ideas works as an indicator which may help firms understand their users' attitudes on the potential innovation that might be applied in their products in future.

In Peterson and Schaefer's literature paper [5], they emphasized the challenge on idea evaluation for social product development in OICs. Being one of the most important characteristics for new ideas, idea popularity has attracted people's attention especially on using it to evaluate new ideas. Previous studies have found that firms use the popularity of ideas to inform internal idea evaluation and make decisions on idea implementation [14, 1]. However, there are conflicted conclusions on whether popularity is appropriate for idea evaluation [15, 16, 17]. This is partly due to the lack of deep understanding on idea popularity, which was emphasized by Peterson and 
Schaefer by stating the necessity of developing deep understanding on it. A number of studies indicated that whether an idea can be popular or not is affected by voter's social behaviors in OICs [18, 19]. Since OICs are operated as social networks for both voters and ideators, a similar influence from ideators' social behaviors on the popularity of their ideas might exist as well. Investigating how the social behaviors of ideators are associated with their idea popularity can help us develop a deep understanding on idea popularity. However, few studies have been focusing on testing this assumption. In order to address this research gap, an empirical study is conducted which collects a large-scale, quantitative data set from the Microsoft online innovation community, including 5468 users, 11985 ideas as well as associated social interaction data between September 2014 (when the platform was launched) and September 2018. We focus on understanding what social behaviors of ideators lead to the high popularity of their ideas. With the analysis of users' online idea posting and commenting behaviors, our results reveal that the overall popularity of a user's ideas is positively related to the contribution of new ideas and the diversity of his/her comments on others' ideas, while negatively related to the number of comments the user had posted. Moreover, user's innovation capability always poses a positive effect on both overall and average popularity of his/her ideas. These findings can help firms better understand idea popularity, and apply it for idea evaluation in order to improve the effectiveness and efficiency of social product development.

\section{Theoretical Background}

OICs as a form of online platforms enable users to post their ideas and review others'. Commenting and voting are two primary forms of activities for users to participate into the reviews of others' ideas [20]. When users find an idea that they like, they can vote to support that idea. The number of votes received primarily reflects the popularity of an idea among its intended users. Since it is important to understand users' preference 
on NPD, idea popularity which is measured by the number of votes received turns out to be a significant characteristic in OICs [14, 1].

Previous studies analytically analyzed the effect of idea popularity on ideas' evaluation and implementation in different OICs. A study [21] conducted on open innovation contest platforms found that votes are used as a measure to determine users' design preferences and pre-select the most promising designs. Further research on an open innovation contest platform named Atizo.com, Hofstetter et al. [14] obtained a similar conclusion that firms use votes to inform internal idea evaluations. Li et al. 22, disclosed that the idea popularity, as well as the ideator's prior participation and prior implementation rate, influence the likelihood of idea implementation. This is consistent with Schemmann et al.[1]'s study on an online idea crowdsourcing platform run by an internationally active beverage producer and retailer. Their analytical results indicated that the popularity of an idea determines whether an idea is implemented or not. However, different conclusions are received in Di Gangi and Wasko's research on Dell's IdeaStorm [23]. More specifically, their analysis did not support the hypothesis that firms would adopt the most popular ideas in the community; indeed, they found that users have a great impact on the popularity of the idea in OICs, which pose pressure on firms' decisions of idea implementation.

Further to the research question about the appropriateness of idea popularity as an indicator for idea evaluation, a number of studies analyzed it from different angles (e.g., [24, 15, 17, 16, 1]), which can be summarized into three areas: studies focusing on business value, on comparison with other evaluation methods, and on customer relationship.

Studies focusing on business value concluded that the popularity of an idea can reflect its commercial attractiveness and market potential. For instance, Hienerth et al. [15] identified a positive relationship between the positive feedback received by a given user-generated design within the peer community and its perceived commercial attractiveness. Furthermore, the study found that the voted popularity of a new product 
idea is often an indicator of its potential acceptance and popularity in the market [25]. These are in line with Schemmann et al.[1]'s study that idea popularity is an important factor for assessing whether an idea is valuable for NPD.

Voted popularity is considered as a pre-selection mechanism which is based on the collective intelligence, or the so-called "wisdom of the crowds" [26]. Previous studies, focusing on popularity as an idea evaluation method, have contrasted it with other methods such as idea evaluation by professional experts. Toubia and Flores [27] found that a large number of consumers in idea screening could be compared with the involvement of a small number of experts when making binary holistic evaluations. Furthermore, it is found that decisions made by a large group of individuals via voting are often better and more precise than decisions made by an elite few [26]. These are consistent with the conclusions from Magnusson et al.'s research [17] about whether users are appropriate substitutes of experts when judging ideas. Their results confirmed that companies can employ users during the initial screening process using criteria assessment to select the best ideas for further elaboration. However, according to [28], the crowd is better suited to deciding simpler aesthetic issues, e.g., the preferred design of a selection of objects, rather than choosing complex technical solutions. Therefore, when it comes to more complex issues, single popular voting is often insufficient with regards to capturing complexity, especially comparing with the evaluation given by experts [28, 29]. Previous studies indicated that the crowd and professional experts have different frames of reference when evaluating how good an idea is. For instance, the crowd does not have to consider issues such as feasibility, or business potential, when assessing an idea [30], while professional experts employed by firms have to take into account strategic issues that may cause the rejection of even very popular ideas (see, for instance, Di Gangi et al.[23]). This indicates that popularity is not an appropriate measure when judging a certain type of ideas.

Studies focusing on customer relationship, found both advantages and disadvantages of taking popularity as an indicator of idea evaluation. Selecting the most popular 
ideas has the advantage of transmitting the message that firms are committed to users' necessities [16]. It helps to build strong relational bonds and goodwill between firms and their intended users [23]. Empowering the crowd to assess new ideas could also have other positive effects such as strengthening a company's competitive advantage by means of their potential customers feeling involved and acknowledged [24]. However, this system requires an explicit and frequent involvement of users who provide ratings on ideas, and studies found that most popular ideas are not always aligned with the company innovation strategic policies [16]. Furthermore, empirical research has shown that rejecting popular ideas may create frustration among the crowd [23].

As discussed above, previous studies have received conflicted results about whether popularity is an appropriate measure for idea evaluation in OICs. This is partly due to the lack of deep understanding on idea popularity. It is therefore of great importance to develop deep understanding on idea popularity, such as how popular ideas are generated and what factors affect the idea popularity. In the study of Dell's IdeasStorm, MartinezTorres [16] found that commenting and voting as the two primary forms of participation in online communities are correlated. Furthermore, Bajic and Lyons [19] disclosed that users favour ideas similar to their own, resulting in more votes and comments on these ideas. This kind of bias on voting has been found in other studies as well. When messages communicate negative feeling, they are likely to attract negative reactions from the community in terms of votes and comments [18].

\section{Model and Hypothesis}

Voting, commenting and posting new ideas are users' main online behaviors in OICs. To measure the influence of ideators' social behaviors on their idea popularity, this study is designed to investigate two types of users' behaviors in OICs which are idea commenting and generating behaviors. Note that the voting behaviors of ideators are not considered in the study because votes cannot be traced back to ideators, thus it is not possible 
to tell which ideator votes which idea. However, the number of votes received by each idea is collected and will be used to measure idea popularity. Two measures related to users' commenting behaviors are adopted, which are the motivation in generating comments and the diversity of generated comments. Further to investigate the users' idea generation behaviors, the motivation in idea generation and the performance on generating implemented ideas are employed in this research. The detailed discussions are presented below, followed by the development of each hypothesis.

Commenting as an important way for people interacting and learning from each other, can generate valuable knowledge and information on NPD [31]. In addition, commenting in OICs serves as a pipe for product-related information sharing among users [32]. Users who acquire such information can develop better understanding of a product, which can promote people's active and critical thinking [33]. Previous research has found that giving attention to others' ideas is an important characteristic for individuals who are successful on innovation [34. This may be due to the fact that exposure to other's creative ideas via commenting can enhance individual's own creativity, which eventually affects the quality and quantity of new ideas [35].

Moreover, commenting on others' ideas also provides unique opportunities for users to interact with each other and build up their social networks. Prior research on creative artists who are widely recognized as lone geniuses has found that embedding in a wider social network significantly helps their innovation development [36]. Users' social networks developed via social behaviors such as commenting in OICs can benefit from the generation of valuable ideas. In addition, expanding their social networks with others via commenting may also attract more attention from others on one's own ideas in OICs. This might lead to an increase in idea popularity, which is reflected by the number of votes users received on their ideas. Therefore, the overall popularity of an individual's ideas which is measured by the total votes s/he received from other users on all of his/her ideas should be positively influenced by his/her motivation in generating comments on others' ideas. 
H1a. The overall popularity of an individual's ideas is positively related to their motivation in generating comments on others' ideas.

Our concern about applying overall votes for measuring idea popularity is that the number of votes an ideator had received might be affected by the number of idea s/he posted. Therefore, it is necessary to employ another indicator to exclude the effects from idea number. In this study, we take the average votes an individual received per idea as a measure of the average popularity of an user's ideas, and offer the following hypothesis:

H1b. The average popularity of an individual's ideas is positively related to their motivation in generating comments on others' ideas.

However, information processing is a time consuming task as information needs to be noted, encoded and interpreted to be useful [37]. Thus, when information is overloaded to people's attention, their behaviors may be constrained by the collected information [38]. More specifically, social behaviors in OICs such as commenting require users to spend much of their time. Also, since users participate into OICs' activities voluntarily, the time they allocate in OICs is limited. The increase of time consumption on commenting may reduce the time allocated on idea generation, which leads to a negative effect on the quality of new ideas people generate. In addition, it might reduce the time users can apply to absorb the information and knowledge they received via interaction in OICs, which may negatively affect the development of their understanding on customers' needs and their ideas on NPD. That is, commenting in OICs may have a negative effect on both overall popularity and average popularity of people's ideas, as it may reduce the quality of ideas they produced. The above discussion is summarized in the following hypotheses:

H1c. The overall popularity of an individual's ideas is negatively related to their motivation in generating comments on others' ideas.

H1d. The average popularity of an individual's ideas is negatively related to their 
motivation in generating comments on others' ideas.

A typical OIC normally includes multiple categories focusing on different functions or aspects of products and services from the host firm. Users are allowed to select the categories they would like to contribute on, via posting, commenting on and voting ideas. The different categories of communities represent different "thought worlds" [39] that expose people to diverse knowledge. Further to users' participation behaviors, some of them choose to be active in only one category while some actively participate in more than one category [40, 30]. It is clear that being active in multiple categories can bring the benefits that allow users access diverse knowledge, so they can transfer, translate and transform knowledge and experiences from one group to another [40].

Prior research has recognized that drawing out knowledge from diverse sources can help people discover and adapt new ideas [41. People who are involved in multiple categories in communities can develop different expertise, which provides them fresh angles for tackling problems and creating solutions [42]. As a main social activity in OICs, commenting on ideas in multiple categories play as a pipe for sharing knowledge and information. Therefore, users who comment in diverse categories are able to develop a better understanding, which leads to the development of high quality ideas that are more likely to be valuable [30]. Moreover, various expertise and good understanding may help ideators generate creative ideas which better meet the needs of customers better. Therefore, ideas generated by people commenting in multiple categories may turn out to be more popular in OICs. Thus, the following hypotheses are offered:

H2a. The overall popularity of an individual's ideas is positively related to the diversity of their comments on others' ideas.

H2b. The average popularity of an individual's ideas is positively related to the diversity of their comments on others' ideas.

Posting new ideas is another type of social activities in OICs. Via posting new ideas, ideators can share their expertise and knowledge with others. Furthermore, 
it creates an opportunity to develop the communication and discussion around new ideas between ideators and other users. Thus, posting new ideas is an important way for ideators to learn from others, particularly about other users' feedback which can enhance ideators' understanding on users' needs. The development of this kind of understanding could lead to ideators' creation of popular ideas. Additionally, launching new ideas may contribute to the expansion of ideators' social networks. People's new ideas may attract the attention of other users to previous ideas they have posted, which may lead to an increase in both the overall and average popularity of people's ideas. In this case, the following hypotheses are offered:

H3a. The overall popularity of an individual's ideas is positively related to their motivation in generating new ideas.

H3b. The average popularity of an individual's ideas is positively related to their motivation in generating new ideas.

Previous research on user involved innovation identified a special group of users, called lead users [43]. Lead users are people who are good at foreseeing customers' demands, and developing valuable suggestions and functional solutions [13, 44]. These studies found that ideas suggested by lead users are more likely to be valuable and possible to be implemented by firms [13]. Furthermore, prior research discovered that lead user-generated ideas tend to attract positive feedback from peers [15]. This may indicate that the ideas generated by lead users normally have good quality and meet users' needs well, and thus attract more support from the rest of users in communities. In addition, lead users are found more active in communities than others, which can enhance the growth of their social networks, attracting more attention on their ideas from other users.

Previous studies have found that voted popularity is applied by firms as a preselection mechanism for evaluating new ideas generated in online communities [29, 30]. This is because the voted popularity of a new idea is an indicator of its potential 
acceptance and success in the market. The number of votes that a new idea received can reflect to what extent the idea is supported by potential customers [25]. Therefore, the implemented ideas in OICs are normally the ones that are popular with peers. Furthermore, for users who generated a greater number of implemented ideas in OICs, they are often considered as ideators who are good at foreseeing customers' demands, and developing valuable suggestions and functional solutions [13, 44]. Therefore, ideas suggested by these users are more likely to attract support from peers and become popular in OICs [15]. This may indicate that not only their implemented ideas but also the rest of their ideas can receive a high number of votes, which can be captured by the overall popularity and the average popularity of their generated ideas in OICs. The following hypotheses summarize the above discussion:

H4a. The overall popularity of an individual's ideas is positively related to their success in generating implemented ideas.

H4b. The average popularity of an individual's ideas is positively related to their success in generating implemented ideas.

\section{Methodology}

\subsection{The dataset}

The study uses publicly available data from an online idea crowdsourcing platform founded by Microsoft, specifically for the Power Business Intelligence (PowerBI) software products. The collected data sample from the platform is suitable to our research as the platform is well established with a large number of users actively contributing to product development. Moreover, the data size, i.e., 11985 records of ideas collected in a duration of four years is appropriate for obtaining insightful results. The platform brings the firm and its users together, collecting ideas directly from PowerBI users on improving existing products and services as well as developing new ones. To partici- 
pate, users join the platform free of charge by creating a profile with an email address. The platform assigns a default username, i.e., anonymous if users do not provide one when accounts are created, however, users are allowed to change their username at a later date. Similar to other popular crowdsourcing platforms, such as Dell IdeaStorm and MyStarBucksIdea, the demographics of users are not collected, and usernames are the identifiers to determine which idea is contributed by whom.

When posting an idea, users are required to give a title and a description, as well as selecting a category that the idea belongs to. There are 19 categories (e.g., Reports, APIs and Embedding, Dashboard, etc.) in the collected data, two new categories have been added after our data collection date. As only 22 ideas (less than $0.14 \%$ of total ideas) fall into the new categories, our data is able to represent the characteristics of whole data. Besides posting ideas, users are able to interact with other users by voting whether they support an idea, and commenting on others' ideas. A user can give maximum one vote to each idea. If a user votes an idea, then the platform shows that the idea receives "1" vote. Thus, the number of votes received by an idea represents how many users have voted for supporting the idea. Note that as usernames are not associated with votes, we are not able to count how many votes a user provided but how many votes each idea had received as the number of votes is recorded at the idea level.

The idea review team of Microsoft makes implementation decisions on the posted ideas in two phases. Firstly, the team reads ideas and identifies the ones that they need to take action on. These identified ideas are assigned with a label to indicate their status. There are eight status categories, including backlog, under review, escalated, planned, started, consideration for backlog, not planned, and completed. Besides the status label, the team provides a comment to the user in order to explain why the label is chosen. The comment may also contain answers to queries if posted together with the idea. In the second phase, the review team works on the labelled ideas and adjusts the status labels if progress has been made. For instance, the 'under review' label of 
Table 1: Descriptive statistics

\begin{tabular}{lcccc}
\hline & Mean & S.D. & Min & Max \\
\hline Number of ideas user contributed $\left(n_{\text {idea }}\right)$ & 4.447 & 6.457 & 1.000 & 45.000 \\
Number of implemented ideas $\left(n_{\text {impl }}\right)$ & 0.286 & 0.8111 & 0.000 & 8.000 \\
Number of comments user provided to others $\left(n_{\text {cmt }}\right)$ & 2.998 & 6.692 & 0.000 & 49.000 \\
Comments diversity $(d i v)$ & 0.139 & 0.239 & 0.000 & 0.982 \\
Number of votes user received on all his/her ideas $\left(y_{\text {oa }}\right)$ & 95.320 & 266.659 & 0.000 & 3137.000 \\
Average number of votes user received per idea $\left(y_{\text {avg }}\right)$ & 21.400 & 57.532 & 0.000 & 972.000 \\
\hline
\end{tabular}

an idea can be replaced by 'started' if the idea is put into implementation process. If an idea gains priority after the review, the label 'escalated' will be assigned. Once the implementation is completed, ideas are assigned with 'completed' status. Unlike Dell IdeaStorm, there is no reward (e.g., money, badge) for users if their ideas are implemented.

The PowerBI platform received its first idea in September 2014. In order to stabilise the interaction around a new idea, we crawled data on all of the ideas posted between September 2014 and June 2018, without collecting ideas posted in the following three months (i.e., July, August, and September 2018). During the data collection period, 11985 ideas were posted, out of which 559 ideas had been implemented. The implementation ratio is $6.0 \%$, which is consistent with other idea crowdsourcing platforms [23. As data on the ideas contributed by users with username anonymous cannot be distinguished from each other, we dropped the ideas posted by anonymous users, allowing the study on the level of individual users. The number of ideas becomes 9243, and 5469 users are involved.

\subsection{Related Measures}

We used measures at the individual level to capture users' idea creation behaviors. The dependent variables measure the popularity of ideas from two aspects: the overall popularity on all the ideas a user had contributed, and the average popularity on individual ideas in general. Observing that count measures have been used to capture 
idea characteristics [14, 1], we measure overall popularity $\left(y_{o a}\right)$ as the total number of votes received by all the ideas which a user had contributed, and average popularity $\left(y_{\text {avg }}\right)$ as the average number of votes user's individual ideas had received.

The measures of user's idea creation behaviors on the PowerBI crowdsourcing platform are based on user's idea posting, voting and commenting behaviors, as well as the implementation status of their post ideas. The independent variable number of comments $\left(n_{c m t}\right)$ is used to measure a user's motivation in generating comments on others' ideas (see H1a - H1d). The value of the variable becomes zero if no comment had been posted by a user during the data collection time period. The independent variable comment diversity (div) captures the diversity of comments on posted ideas over different idea categories (see H2a, H2b). We use Shannon entropy which has been exploited as a diversity measure for a number of applications [45, 30], to measure the independent variable comment diversity as an entropy over the 19 idea categories. More formally, we calculate the comment diversity as $-\sum_{j=1}^{n} p_{j} \log p_{j}$, where $p_{j}$ is the proportion of the number of comments posted by an user in category $j$ to the total number of comments posted by the user, and $n$ is the number of idea categories. We have $n=19$ in this study. The entropy measure calculates the comment diversity into a range of $[0,1]$, and the greater the value, the more diverse in terms of users commenting behaviors. The number of ideas $\left(n_{\text {idea }}\right)$ a user had contributed is a measure for the motivation in generating new ideas (see H3a and H3b). We use the total number of ideas which were contributed by a user and had been implemented by Microsoft (denoted by $n_{\text {impl }}$ ), to measure a user's success in generating implemented ideas. As it can take some time to complete the implementation of an idea in practice, the implementation status of an idea is coded as 1 if the status label assigned by Microsoft review team is 'completed', 'started', or 'escalated'; otherwise, it is coded as 0 . Thus the independent variable $n_{i m p l}$ takes the sum of the implementation status on all the ideas the user had posted.

Table 1 provides the descriptive statistics for the dependent and independent variables. In our data, a user contributed an average of 4.447 ideas and the maximum 
number of ideas by a single user was 45. Among these contributed ideas, 0.286 ideas were implemented on average while the maximum was 8 and minimum was zero. The standard deviation for the number of contributed ideas and implemented ideas by a single user are 6.457 and 0.8111 respectively (see Table 1), indicating that the data points of both measures are close to their mean and for the number of implemented ideas, the data points are closer to the mean at 0.286 . Thus, most users have only few posted ideas that have been successfully implemented. In terms of the commenting behaviors, an average of 2.998 comments were generated per user and the maximum was 49. The comments diversity on average was 0.139 and the maximum was 0.982 . The low average value of comments diversity indicates that users posted comments in limited number of idea categories. Regarding the popularity of ideas, the number of votes received on all the ideas a user had posted was 95.320 on average and its maximum was 3137. Moreover, the average number of votes received per idea was 21.400 with the maximum at 972 . The standard deviation for these two popularity measures are 266.659 and 57.532 respectively (see Table 1), indicating that the data points for both measures are spread out over a large range of values. Therefore, the popularity of a user's ideas varies.

We first look at the correlative evidence for our hypotheses. We used the Pearson correlation test for all the measures by assuming linear relations among the measures. As shown in Table 2, the dependent variable overall popularity has significant relationship with all the independent variables $(p<.001)$. More specifically, the strongest positive correlation is detected between overall popularity and the number of implemented ideas $(r=.6306, p<.001)$. The correlation between overall popularity and the number of ideas is also positive $(r=.4048, p<.001)$, which is stronger than the relationship with the number of comments and comments diversity $(r=.2870, p<.001$; $r=.2748, p<.001)$.

The correlation test for the other dependent variable, average popularity shows that it has no relationship with the number of comments, comments diversity and number 
Table 2: Correlations

\begin{tabular}{|c|c|c|c|c|c|c|}
\hline & Overall popularity & Average popularity & Number of comments & Comments diversity & Number of ideas & Number of implemented ideas \\
\hline Overall popularity & 1 & & & & & \\
\hline Average popularity & $0.5620^{* * *}$ & 1 & & & & \\
\hline Number of comments & $0.2870^{* * *}$ & -0.0039 & 1 & & & \\
\hline Comments diversity & $0.2748^{* * *}$ & 0.0054 & $0.7844^{* * *}$ & 1 & & \\
\hline Number of ideas & $0.4048^{* * *}$ & 0.0004 & $0.7959^{* * *}$ & $0.6994^{* * * *}$ & 1 & \\
\hline Number of implemented ideas & $0.6306^{* * *}$ & $0.2396^{* * *}$ & $0.3188^{* * *}$ & $0.2817^{* * *}$ & $0.4752^{* * *}$ & 1 \\
\hline
\end{tabular}

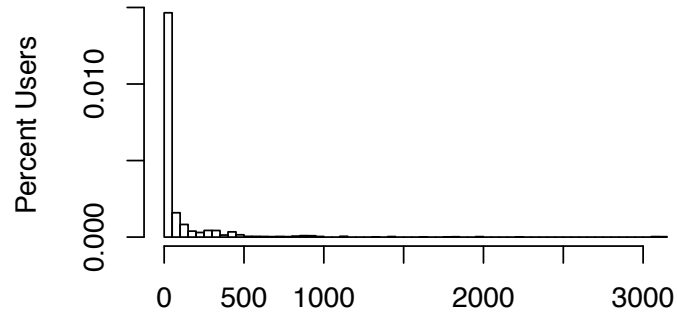

Number of votes user received on all his/her ideas

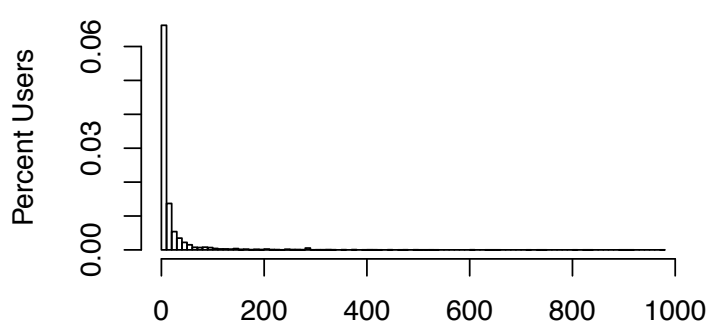

Average number of votes user received per idea

(a) Distribution of the number of votes re- (b) Distribution of the average number of

ceived on all ideas

votes received per idea

Figure 1: Distribution of the dependent variables

of ideas, but significantly relates to the number of implemented ideas $(r=.2396$, $p<.001)$.

\section{$5 \quad$ Findings}

The descriptive statistics (as displayed in Table 1) indicate that there is overdispersion in both dependent variables, i.e., overall popularity and average popularity. This is because the values of standard deviation which represents the variance of the distribution of these two variables are both much greater than the mean. We plot the distribution of the two variables in Figure 1 to show the overdispersion: the two variables respectively has a count ranging from 0 to 3137 and to 972 respectively with mass at low values. In order to accommodate for the count nature of the dependent variables as well as the data overdispersion, we use a negative binomial regression approach [46. Eq. 1 illustrates the negative binomial model (Model 1) where the overall popularity $y_{o a}$ is the dependent variable. 


$$
\log (\mu)=\alpha+\beta_{1} n_{c m t}+\beta_{2} d i v+\beta_{3} n_{i d e a}+\beta_{4} n_{i m p l}
$$

where $P\left(Y=y_{o a} \mid n_{c m t}\right.$, div, $\left.n_{\text {idea }}, n_{\text {impl }}, k\right)=\frac{\tau\left(y_{o a}+k\right)}{\tau(k) \tau\left(y_{o a}+1\right)}\left(\frac{k}{k+\mu}\right)^{k}\left(\frac{\mu}{k+\mu}\right)^{y_{o a}}$, and $E(Y)=\mu, V(Y)=\mu+\left(\mu^{2} / k\right)$.

As shown in Table 3, the relationship between overall popularity $y_{o a}$ and number of comments user contributed $n_{c m t}$ is significant and negative $(-0.0376)$, which is in accordance with hypothesis H1c. The diversity of comments (div), number of ideas $\left(n_{\text {idea }}\right)$ and number of implemented ideas $\left(n_{\text {impl }}\right)$ are all positively associated with the overall popularity $\left(\beta_{2}=1.8045, \beta_{3}=0.0824, \beta_{4}=0.8088, p<.001\right)$. These findings are in line with hypotheses H2a, H3a and H4a. We find that the coefficient of the number of implemented ideas is greater than the number of ideas, indicating that the influence of success in generating implemented ideas might be stronger on the idea popularity. In order to test whether the negative binomial model deals with the overdispersion well, we conduct the Pearson's chi-squared test which compares Model 1 with a Poisson model (i.e., Model 2). As shown in Table 3, the Poisson model outputs similar results. However, the chi-squared value in the Poisson model is much larger than our Model 1 (see Table 5). As the dispersion parameter of the Poisson model is also larger than Model 1, we can conclude that Model 1 fits the data better than Model 2. Therefore, H1c, H2a, H3a and H4a are supported.

Further, we continue using the negative binomial approach to test the hypotheses with the dependent variable, average popularity $\left(y_{\text {avg }}\right)$. We replace $y_{\text {avg }}$ in Eq. 1 with $y_{\text {avg }}$ to get the following equation which forms Model 3:

$$
\log (\mu)=\alpha+\beta_{1} n_{c m t}+\beta_{2} d i v+\beta_{3} n_{i d e a}+\beta_{4} n_{i m p l}
$$

where $P\left(Y=y_{\text {avg }} \mid n_{c m t}\right.$, div, $\left.n_{\text {idea }}, n_{\text {impl }}, k\right)=\frac{\tau\left(y_{\text {avg }}+k\right)}{\tau(k) \tau\left(y_{\text {avg }}+1\right)}\left(\frac{k}{k+\mu}\right)^{k}\left(\frac{\mu}{k+\mu}\right)^{y_{\text {avg }}}$, and $E(Y)=\mu, V(Y)=\mu+\left(\mu^{2} / k\right)$. 
The regression results are shown in Table 4. More specifically, the number of comments has no significant relationship with the average popularity $(p>.1)$. This finding rejects both $\mathrm{H} 1 \mathrm{~b}$ and $\mathrm{H} 1 \mathrm{~d}$.

However, comments diversity is positively related to average popularity $\left(\beta_{2}=\right.$ $0.7131, p<.001)$. The number of ideas has a negative relationship with the average popularity at significant level of $p<.001$. Moreover, the number of implemented ideas is positively related to the average popularity $\left(\beta_{4}=0.7276, p<.001\right)$. Similar to the comparison between Model 1 and 2, we conduct Pearson's chi-squared test for Model 3 against a Poisson model, i.e., Model 4. As displayed in Table 5, Model 3 fits the data better because of the low chi-squared value and dispersion parameter.

Although the negative binomial model shows a significant relationship between average popularity and comments diversity as well as the number of ideas, the correlation analysis (Table 2) indicates they are not correlated. To further test the relationship, we develop two negative binomial models: each includes only one of these two independent variables (see Model 5 and 6 in Table 4). The results of both models confirm the correlation analysis that comments diversity and number of ideas are not significantly related to the average popularity. The VIF test for identifying multicollinearity issues in the independent variables suggests the correlation among independent variables is acceptable with VIF smaller than 4 [47]. The findings indicate that comments diversity and number of ideas might be suppressor variables which are correlated with one or more independent variables to suppress irrelevant variance of independent variables [48]. We then construct a negative binomial model (see Model 7 in Table 4) which includes only the number of implemented ideas to test the suppressing influence. The result shows that the number of implemented ideas is positively related to average popularity, and the coefficient decreases from 0.7276 in Model 3 to 0.5753 in Model 7. This finding indicates that by including comments diversity and number of ideas in the regression model (i.e., Model 3), it helps to strengthen the effect of the number of implemented ideas. Therefore, $\mathrm{H} 2 \mathrm{~b}$ and $\mathrm{H} 3 \mathrm{~b}$ are rejected but $\mathrm{H} 4 \mathrm{~b}$ is supported. 
Table 3: Regression results on H1a - H4a, H1c

\begin{tabular}{lllll}
\hline & \multicolumn{2}{c}{ Model 1 (NB) } & \multicolumn{2}{c}{ Model 2 (Poisson) } \\
Variable & Coeff. & SE & Coeff. & SE \\
\hline Constant & $3.1289^{* * *}$ & 0.0180 & $3.6835^{* * *}$ & 0.0017 \\
$n_{\text {cmt }}:$ number of comments & $-0.0376^{* * *}$ & 0.0042 & $-0.0053^{* * *}$ & 0.0002 \\
div: comments diversity & $1.8045^{* * *}$ & 0.0982 & $1.5681^{* * *}$ & 0.0055 \\
$n_{\text {idea }}:$ number of ideas & $0.0824^{* * *}$ & 0.0040 & $0.0182^{* * *}$ & 0.0002 \\
$n_{\text {impl }}:$ number of implemented ideas & $0.8088^{* * *}$ & 0.0201 & $0.5202^{* * *}$ & 0.0006 \\
\hline
\end{tabular}

***: p-value $<0.001 ; * *$ p-value $<0.01 ;{ }^{*}$ : p-value $<0.05$

NB represents negative binomial.

Table 4: Regression results on H1b - H4b, H1d

\begin{tabular}{|c|c|c|c|c|c|c|c|c|c|c|}
\hline \multirow[b]{2}{*}{ Variable } & \multicolumn{2}{|c|}{ Model $3(\mathrm{NB})$} & \multicolumn{2}{|c|}{ Model 4 (Poisson) } & \multicolumn{2}{|c|}{ Model 5 (NB) } & \multicolumn{2}{|c|}{ Model 6 (NB) } & \multicolumn{2}{|c|}{ Model 7 (NB) } \\
\hline & Coeff. & $\mathrm{SE}$ & Coeff. & SE & Coeff. & $\mathrm{SE}$ & Coeff. & $\mathrm{SE}$ & Coeff. & SE \\
\hline Constant & $2.9070^{* * *}$ & 0.0178 & $3.0519^{* * *}$ & 0.0029 & $3.0544^{* * *}$ & 0.0173 & $3.0622^{* * *}$ & 0.0182 & $2.7844^{\text {*** }}$ & 0.0153 \\
\hline$n_{c m t}:$ number of comments & 0.0012 & 0.0041 & $0.0161^{* * *}$ & 0.0006 & & & & & & \\
\hline div: comments diversity & $0.7131^{* * *}$ & 0.0976 & $0.5360^{* * *}$ & 0.0146 & 0.0650 & 0.0625 & & & & \\
\hline$n_{\text {idea }}:$ number of ideas & $-0.0691^{* * *}$ & 0.0040 & $-0.0824^{* * *}$ & 0.0008 & & & 0.0003 & 0.0023 & & \\
\hline$n_{\text {impl: }}$ number of implemented ideas & $0.7276^{* * *}$ & 0.0199 & $0.5117^{* * *}$ & 0.0018 & & & & & $0.5753^{* * *}$ & 0.0176 \\
\hline
\end{tabular}

Table 5: Pearson chi-squared tests for selected models

\begin{tabular}{lllll}
\hline & Model 1 (NB) & Model 2 (Poisson) & Model 3 (NB) & Model 4 (Poisson) \\
\hline Pearson chi-squared & 33989.3103 & 1180327.5274 & 25548.8906 & 2538706.2068 \\
dispersion & 3.6247 & 125.8747 & 2.7246 & 270.7376 \\
\hline
\end{tabular}

NB represents negative binomial.

\section{Discussion}

In this study, the relation between idea popularity and ideators' social behavior in OICs is empirically investigated. Five years of large-scale data involving thousands of ideas and users are studied in the context of Microsoft's OIC. The Microsoft's OIC allows users to contribute on their NPD via posting, commenting on and voting ideas. The number of votes an individual's ideas received from other users represents how popular the individual's ideas are. Both the overall popularity and the average popularity of an individual's ideas are studied in this research. Furthermore, commenting on others' ideas and posting users' own ideas serve as the main social behaviors in OICs. To measure users' commenting behavior, the motivation and the diversity of comments are adopted as indicators in this study. Further to users' idea creation behavior, it 
is measured by the motivation in generating new ideas and the success in generating implemented ideas.

Our research disclosed a complicated relationship between the overall idea popularity and commenting behavior. The overall popularity of an individual's ideas is found negatively related to the motivation of his/her comments which is measured by the number of comments the user gave to others' ideas, but positively related to the diversity of comments which is captured by an entropy measure on the number of categories the user commented on. These results disrupted the former understanding in the research area. Previous studies found that being active in communities by giving more comments and interactions can benefit the development of people's knowledge and expertise, together with the growth of their social networks [36, 35]. It is more likely for people who benefit from it to create high-quality ideas, which also turn out to be more popular in communities as they can meet the demand of intended customers better. The negative relationship between the overall popularity and comment motivation found in this research indicates that the users experiencing high comment motivation contribute ideas with low overall popularity while the users receiving high overall popularity of their ideas have low comment motivation. One possible explanation is that different users take different roles and activities in OICs. The main purpose for firms setting up their OICs is to source valuable new ideas from their users. However, as a social community with its own ecosystem, a successful OIC does not operate based only on new idea generation. As we discussed before, activities including commenting and voting play an important role for the generation and selection of valuable new ideas in OICs. Our research results may provide evidence demonstrating that users in OICs have their own preference for participating into activities, i.e., generating, commenting on and voting ideas. Users have the freedom to choose whether they would like to contribute more on generating ideas or commenting on others' ideas. In this research, a positive relation is found between the number of generated new ideas and the overall popularity of an individual's ideas. Therefore we can conclude that users who focus 
their efforts on idea generation have more chances of achieving high overall popularity with their ideas. Alternatively, users who are more engaged with idea commenting may experience low overall popularity, This could explain the fact they are spending more time commenting on ideas as opposed to generating them. This can also be applied to explain why there is a positive relation between the overall popularity and comment diversity. An increase in an individual's comment diversity may suggest an increase in the number of posted ideas, as different categories of communities represent different "thought worlds" 39]. The "thought worlds" expose people to diverse creation opportunities. In fact, a positive relation between the overall popularity of an individual's ideas and his/her idea creation behavior has been confirmed in this research. Both the motivation in generating new ideas and the success in generating implemented ideas are positively related to the overall popularity of people's ideas. The findings indicate that users who create more ideas, especially ideas implemented by firms, would experience more votes from other users. As we explained above, it may be because the increase of the number of generated ideas provides more opportunities for ideators to receive votes from others. In summary, overall popularity is positively related to idea creation behavior and comment diversity, but has negative relation with comment motivation.

The average popularity which is measured by the average number of votes a user received for each idea is also studied in this research. The result does not identify any relationship between the average popularity and comment motivation, which suggests that giving comments to others' ideas does not show any effect on the average popularity. Furthermore, a suppression relationship was found between average popularity and comment diversity, which is aligned with the relationship between average popularity and comment motivation. In general, commenting behavior does not present significant effects on the average popularity of people's ideas. Previous studies believe that having interaction with each other via commenting in communities is able to help users develop ideas which become more popular in crowd [35]. However, our research does not find supporting evidence for the average level of idea popularity. Furthermore, 
interesting results are obtained on the relation between idea creation behavior and average popularity. There is a suppression relationship between average popularity and the motivation of generating new ideas, while a positive relation is found between average popularity and the success in generating implemented ideas. These results suggest that the average popularity of an individual's ideas is not affected by how many new ideas a user generated but by how many implemented ideas s/he posted. Therefore, on average level, how popular an individual's ideas is not related to the number of posted ideas; instead, it is related to the quality of ideas which is captured by whether the ideas are implemented or not.

\section{Implications}

This research is an important step towards addressing the question of how idea popularity in OICs can be applied to stimulate innovation on social product development. Our study finds a positive relationship between the popularity of people's ideas and their success in generating implemented ideas. This result provides new evidence to understand whether idea popularity can be applied to evaluate ideas in OICs. Our finding is consistent with the conclusion of "wisdom of the crowds" which supports the application of voted popularity as a pre-selection mechanism for the implementation of ideas in communities [26]. Considering the huge number of new ideas generated in OICs, applying voted popularity as a pre-selection mechanism can help firms save time and human resources that they need to invest into idea selection.

Previous concern about using voted popularity as a pre-selection mechanism is that users can be biased towards voting [19, 18]. People's behaviors such as voting might be affected by their social networks. For example, an ideator who has frequent interactions and strong connections with others may attract more users to vote his/her ideas. Therefore, being biased towards voting would reduce the effectiveness of using popularity as a pre-selection mechanism for idea evaluation. Indeed, our research 
finds that social behaviors including idea creation and comment diversity are positively related to the overall popularity of an individual's ideas. However, no relation is found between the average popularity and the commenting behaviors as well as the motivation of idea generation. These findings indicate that an ideator's social behaviors may affect the overall votes $\mathrm{s} /$ he receives on all of his/her ideas, but not on the average votes. Moreover, the effect observed on the overall popularity of an individual's ideas may be triggered by the social behaviors related to the creation of new ideas. In other words, social behaviors may stimulate the increase of the number of new ideas which create more opportunities to receive votes from others.

Furthermore, this study demonstrates that different users play different roles and participate in different activities in OICs. Our results suggest that not all users in OICs focus on posting new ideas with high popularity. Although some users put great effort to generate valuable new ideas, other users in OICs are more interested in commenting and voting others' ideas [20]. The users who contribute more valuable ideas are often called lead users [43]. This is a group of users who are recognized in literature as the people who have the ability to provide valuable suggestions and develop functional solutions [13, 44]. Moreover, lead users are generally more active in the community, and have been found to actively participate into commenting and discussing other users' ideas [44, 49], and their ideas are usually more popular in communities. Our finding on the positive relationship between average popularity and the success of generating implemented ideas is aligned with other studies. However, no relation was identified between average popularity and commenting behavior, suggesting that creating popular ideas and being active within community are not related. This finding is useful with regard to the process of identifying lead users in OICs. Thus, our study contributes to the understanding of the characteristics of lead users, which can be used to better identify and analyze lead users in OICs.

Besides lead users, our research may help to reveal the existence of other types of users in OICs. There are users who might not make an equal contribution on idea 
generation as lead users do, but contribute significantly on idea evaluation and idea discussion [20]. In fact, both idea evaluation and idea discussion create important values for OICs. As OICs are utilised by firms to source valuable ideas from users, having users actively commenting and voting ideas can encourage OICs to benefit from the "wisdom of the crowds", which has been used as a pre-selection mechanism for the implementation of ideas [26]. Because searching valuable ideas from a huge number of ideas is a time costly job, having users participate in the process of idea evaluation and selection can significantly reduce the time firms spend on the process [17]. Moreover, adopting users' comments and votes as a pre-selection mechanism has been demonstrated as an appropriate substitute of involving experts on judging ideas [17, 27]. Apart from idea evaluation, users' comments may also serve as a channel for the interaction between ideators and peers, which can help ideators better understand existing product problems and develop better solutions [20]. Thus, an OIC cannot achieve its success without the contribution from this group of users who contribute to commenting and voting. Therefore, firms should bear this in mind when designing their OICs. An efficient mechanism should be created in OICs for stimulating various contributions from different users on different activities including generating, commenting and voting ideas.

\section{Conclusion}

Firms have becoming more and more interested in the adoption of social product development strategies, such as mass collaboration and crowdsourcing. This is due to the fact that consumers who have specialized knowledge about firms' products are found intrinsically motivated to freely contribute their ideas for NPD [1]. Additionally, the development of social computing technologies makes it possible for firms to involve a crowd of users in the NPD. OICs as a popular application of such technologies have been widely used by large firms to collect ideas from their users and facilitate the 
product development process. While it is extensively studied that product ideas with high popularity on OICs are of great importance to NPD, research that explores how the social behaviours of online users affects high popularity is largely unclear. This paper conducts an empirical study by collecting a large-scale, quantitative data set from an OIC between 2014 and 2018. With the analysis of users' online idea posting and commenting behaviors, our results reveal that the idea contribution experience, together with the diversity of comments on other users' ideas positively influence the overall popularity of an individual's ideas, while the motivation of providing comments is negatively related to the overall popularity. Moreover, user's innovation capability poses a positive effect on both overall and average popularity of user' ideas; however, the idea contribution experience, motivation of providing comments as well as comments diversity do not have significant relationship with the average popularity. These findings can help firms better incentivize their users on OICs by applying appropriate strategies to different social behaviors in order to improve the effectiveness and efficiency of social product development.

When interpreting the results of our study, certain limitations need to be kept in mind. Users' motivation and diversity in generating comments are selected as comment characteristics in this study, whilst other characteristics may be associated with individuals' idea popularity, such as comment quality and comment content. User's comments can be categorized through the use of an automated text-mining method in order to identify more detailed characteristics, which may lead to interesting contingency effects. While the static approach is acceptable for examining the research questions, the use of a longitudinal analytical approach may disclose more comprehensive results, such as how users' idea popularity changes along with their continuous social behaviors on OICs. In addition, only one type of crowdsourcing platform is studied in this research, future studies could extend our knowledge by investigating the relationship of users' social behavior and idea popularity in different crowdsourcing contexts. 


\section{References}

[1] B. Schemmann, A. M. Herrmann, M. M. Chappin, and G. J. Heimeriks, "Crowdsourcing ideas: Involving ordinary users in the ideation phase of new product development," Research Policy, vol. 45, no. 6, pp. 1145-1154, 2016.

[2] H. Chesbrough, W. Vanhaverbeke, and J. West, Open innovation: Researching a new paradigm. Oxford University Press on Demand, 2006.

[3] V. Van de Vrande, J. P. De Jong, W. Vanhaverbeke, and M. De Rochemont, "Open innovation in smes: Trends, motives and management challenges," Technovation, vol. 29, no. 6-7, pp. 423-437, 2009.

[4] D. Schaefer, "Industrial game changers for the socio-sphere," 2012.

[5] A. Peterson and D. Schaefer, "Social product development: introduction, overview, and current status," in Product Development in the Socio-sphere. Springer, 2014, pp. 1-33.

[6] E. Von Hippel, "Democratizing innovation: The evolving phenomenon of user innovation," Journal für Betriebswirtschaft, vol. 55, no. 1, pp. 63-78, 2005.

[7] G. Castellion and S. K. Markham, "Perspective: New product failure rates: Influence of a rgumentum ad p opulum and self-interest," Journal of Product Innovation Management, vol. 30, no. 5, pp. 976-979, 2013.

[8] D. J. Flint, "Compressing new product success-to-success cycle time: Deep customer value understanding and idea generation," Industrial marketing management, vol. 31, no. 4, pp. 305-315, 2002.

[9] J. Howe, "The rise of crowdsourcing," Wired magazine, vol. 14, no. 6, pp. 1-4, 2006 .

[10] K. Abhari, E. J. Davidson, and B. Xiao, "Measuring the perceived functional affordances of collaborative innovation networks in social product development," in 
2016 49th Hawaii International Conference on System Sciences (HICSS). IEEE, 2016, pp. 929-938.

[11] D. Wu, D. W. Rosen, J. H. Panchal, and D. Schaefer, "Understanding communication and collaboration in social product development through social network analysis," Journal of Computing and Information Science in Engineering, vol. 16, no. 1, p. 011001, 2016.

[12] F. T. Piller, A. Vossen, and C. Ihl, "From social media to social product development: the impact of social media on co-creation of innovation," Die Unternehmung, vol. 65, no. 1, 2012.

[13] D. Mahr and A. Lievens, "Virtual lead user communities: Drivers of knowledge creation for innovation," Research policy, vol. 41, no. 1, pp. 167-177, 2012.

[14] R. Hofstetter, S. Aryobsei, and A. Herrmann, "Should you really produce what consumers like online? empirical evidence for reciprocal voting in open innovation contests," Journal of Product Innovation Management, vol. 35, no. 2, pp. 209-229, 2018.

[15] C. Hienerth, E. Von Hippel, and M. B. Jensen, "User community vs. producer innovation development efficiency: A first empirical study," Research policy, vol. 43, no. 1, pp. 190-201, 2014.

[16] M. R. Martínez-Torres, "Analysis of open innovation communities from the perspective of social network analysis," Technology Analysis 8 Strategic Management, vol. 26 , no. 4 , pp. $435-451,2014$.

[17] P. R. Magnusson, E. Wästlund, and J. Netz, "Exploring users' appropriateness as a proxy for experts when screening new product/service ideas," Journal of Product Innovation Management, vol. 33, no. 1, pp. 4-18, 2016.

[18] S. Kim and S. Miranda, "Seeds of change: Substance and influence in brand communities," 2011. 
[19] D. Bajic and K. Lyons, "Leveraging social media to gather user feedback for software development," in Proceedings of the 2nd international workshop on Web 2.0 for software engineering. ACM, 2011, pp. 1-6.

[20] T. Ogink and J. Q. Dong, "Stimulating innovation by user feedback on social media: The case of an online user innovation community," Technological Forecasting and Social Change, 2017.

[21] J. Füller, "Refining virtual co-creation from a consumer perspective," California management review, vol. 52, no. 2, pp. 98-122, 2010.

[22] M. Li, A. Kankanhalli, and S. H. Kim, "Which ideas are more likely to be implemented in online user innovation communities? an empirical analysis," Decision Support Systems, vol. 84, pp. 28-40, 2016.

[23] P. M. Di Gangi and M. Wasko, "Steal my idea! organizational adoption of user innovations from a user innovation community: A case study of dell ideastorm," Decision Support Systems, vol. 48, no. 1, pp. 303-312, 2009.

[24] C. Fuchs and M. Schreier, "Customer empowerment in new product development," Journal of product innovation management, vol. 28, no. 1, pp. 17-32, 2011.

[25] H. J. Schau, A. M. Muñiz Jr, and E. J. Arnould, "How brand community practices create value," Journal of marketing, vol. 73, no. 5, pp. 30-51, 2009.

[26] J. Surowiecki, "The wisdom of crowds: Why the many are smarter than the few and how collective wisdom shapes business," Economies, Societies and Nations, vol. 296,2004 .

[27] O. Toubia and L. Florès, "Adaptive idea screening using consumers," Marketing Science, vol. 26, no. 3, pp. 342-360, 2007.

[28] G. P. Pisano and R. Verganti, "Which kind of collaboration is right for you," Harvard business review, vol. 86, no. 12, pp. 78-86, 2008. 
[29] C. Riedl, I. Blohm, J. M. Leimeister, and H. Krcmar, "The effect of rating scales on decision quality and user attitudes in online innovation communities," International Journal of Electronic Commerce, vol. 17, no. 3, pp. 7-36, 2013.

[30] B. L. Bayus, "Crowdsourcing new product ideas over time: An analysis of the dell ideastorm community," Management science, vol. 59, no. 1, pp. 226-244, 2013.

[31] F. T. Rothaermel and S. Sugiyama, "Virtual internet communities and commercial success: individual and community-level theory grounded in the atypical case of timezone. com," Journal of management, vol. 27, no. 3, pp. 297-312, 2001.

[32] J. M. Podolny, "Networks as the pipes and prisms of the market," American journal of sociology, vol. 107, no. 1, pp. 33-60, 2001.

[33] T. Anderson, R. Liam, D. R. Garrison, and W. Archer, "Assessing teaching presence in a computer conferencing context," 2001.

[34] A. C. Bullinger, A.-K. Neyer, M. Rass, and K. M. Moeslein, "Community-based innovation contests: Where competition meets cooperation," Creativity and innovation management, vol. 19, no. 3, pp. 290-303, 2010.

[35] B. A. Nijstad and W. Stroebe, "How the group affects the mind: A cognitive model of idea generation in groups," Personality and social psychology review, vol. 10, no. 3, pp. 186-213, 2006.

[36] B. Uzzi and J. Spiro, "Collaboration and creativity: The small world problem," American journal of sociology, vol. 111, no. 2, pp. 447-504, 2005.

[37] L. Dahlander and S. O'Mahony, "Progressing to the center: Coordinating project work," Organization science, vol. 22, no. 4, pp. 961-979, 2011.

[38] B. Uzzi, "Social structure and competition in interfirm networks: The paradox of embeddedness," Administrative science quarterly, pp. 35-67, 1997.

[39] D. Dougherty, "Interpretive barriers to successful product innovation in large firms," Organization science, vol. 3, no. 2, pp. 179-202, 1992. 
[40] P. R. Carlile, "Transferring, translating, and transforming: An integrative framework for managing knowledge across boundaries," Organization science, vol. 15, no. 5, pp. 555-568, 2004.

[41] L. B. Jeppesen and K. R. Lakhani, "Marginality and problem-solving effectiveness in broadcast search," Organization science, vol. 21, no. 5, pp. 1016-1033, 2010.

[42] R. S. Burt et al., Brokerage and closure: An introduction to social capital. Oxford university press, 2005.

[43] E. Von Hippel, "Lead users: a source of novel product concepts," Management science, vol. 32, no. 7, pp. 791-805, 1986.

[44] P. D. Morrison, J. H. Roberts, and D. F. Midgley, "The nature of lead users and measurement of leading edge status," Research policy, vol. 33, no. 2, pp. 351-362, 2004 .

[45] D. A. Harrison and K. J. Klein, "What's the difference? diversity constructs as separation, variety, or disparity in organizations," Academy of management review, vol. 32, no. 4, pp. 1199-1228, 2007.

[46] A. C. Cameron and P. K. Trivedi, Regression analysis of count data. Cambridge university press, 2013, vol. 53 .

[47] C. M. Ringle, S. Wende, and J.-M. Becker, "Smartpls 3. boenningstedt: Smartpls gmbh," 2015.

[48] R. L. Smith, J. W. Ager Jr, and D. L. Williams, "Suppressor variables in multiple regression/correlation," Educational and Psychological measurement, vol. 52, no. 1, pp. 17-29, 1992.

[49] G. L. Urban and E. Von Hippel, "Lead user analyses for the development of new industrial products," Management science, vol. 34, no. 5, pp. 569-582, 1988. 